\title{
Degenerate four-wave-mixing spectroscopy on epitaxially laterally overgrown GaN: Signals from below the fundamental absorption edge
}

\section{$\operatorname{AUTHOR}(S):$}

Omae, K; Kawakami, Y; Fujita, S; Kiyoku, Y; Mukai, T

\section{CITATION:}

Omae, K ... [et al]. Degenerate four-wave-mixing spectroscopy on epitaxially laterally overgrown GaN: Signals from below the fundamental absorption edge. APPLIED PHYSICS LETTERS 2001, 79(15): 2351-2353

\section{ISSUE DATE:}

2001-10-08

URL:

http://hdl.handle.net/2433/50146

\section{RIGHT:}

Copyright 2001 American Institute of Physics. This article may be downloaded for personal use only. Any other use requires prior permission of the author and the American Institute of Physics. 


\title{
Degenerate four-wave-mixing spectroscopy on epitaxially laterally overgrown GaN: Signals from below the fundamental absorption edge
}

\author{
Kunimichi Omae, ${ }^{\text {a) }}$ Yoichi Kawakami, and Shigeo Fujita \\ Department of Electronic Science and Engineering, Kyoto University, Kyoto 606-8501, Japan
}

Yukihiro Kiyoku and Takashi Mukai

Nitride Semiconductor Research Laboratory, Nichia Corporation, 491 Oka, Kaminaka, Anan, Tokushima 774-8601, Japan

(Received 13 March 2001; accepted for publication 24 July 2001)

\begin{abstract}
We performed photoluminescence (PL) and degenerate four-wave-mixing (FWM) spectroscopy in epitaxially laterally overgrown $\mathrm{GaN}$ at $10 \mathrm{~K}$. Optical transitions based on exciton complexes such as biexciton emission, exciton-exciton scattering, electron-hole plasma, and so on, were revealed by PL under a wide range of excitation densities. The FWM signals were observed from states below the fundamental excitonic absorption edge, showing that nonlinear photoswitching can be performed with a transmittance geometry. The origin of such nonlinearlity was discussed by correlating with feasible many-body effects between excitons, biexcitons, and free carriers. (C) 2001 American Institute of Physics. [DOI: 10.1063/1.1407298]
\end{abstract}

A number of breakthroughs in the fabrication technology of GaN-based semiconductors have opened the way to the commercialization of laser diodes, as well as of lightemitting diodes. Such progress has also contributed detailed studies on linear and nonlinear spectroscopy of excitonic transitions in hexagonal $\mathrm{GaN}(h-\mathrm{GaN})$ epilayers with relatively high optical quality. ${ }^{1-3}$ Free excitons in $h-\mathrm{GaN}$ are composed of three bands labeled $E_{X A}$ (A exciton), $E_{X B}(B$ exciton) and $E_{X C}(C$ exciton), which are transitions from the conduction $\left(\Gamma_{7 c}\right)$ to the $A\left(\Gamma_{9 v}\right), B\left(\Gamma_{7 u v}\right)$, and $C\left(\Gamma_{7 l v}\right)$ valence bands, respectively. The binding energy $\left(E_{\mathrm{ex}}\right)$ and the effective Bohr radius $\left(r_{B}\right)$ of the $A$ exciton $\left(E_{X A}\right)$ in $h$-GaN have been reported to be about $28 \mathrm{meV}$ and about $2.9 \mathrm{~nm}$, respectively. ${ }^{4}$ Fisher et al. performed transient four-wavemixing (FWM) studies on a $\mathrm{GaN}$ epilayer and observed quantum beating between the $A$ and $B$ excitons. ${ }^{1}$ Furthermore, Zimmermann et al. discussed the beating period and the power spectrum of FWM revealed the energy splitting of $7.98 \mathrm{meV}$ between the $A$ and $B$ excitons, and the biexciton binding energy $\left(E_{b}\right)$ of $5.7 \mathrm{meV}^{2}$ Aoki et al. reported that the exciton-exciton interaction plays a major role in the quantum beat process in $\mathrm{GaN}$ by employing polarization dependence in the FWM experiment. ${ }^{3}$ So far, FWM studies have focused on the energy range of intrinsic excitonic resonances under weak excitation conditions. In this letter, we observed FWM signals in epitaxially laterally overgrown (ELO) $\mathrm{GaN}$ from the states under the band-gap energy where almost no optical absorption is observed in linear spectroscopy. Although FWM signals below the band gap have already been observed in InP, ${ }^{5}$ below-gap nonlinearlity peculiar to GaN was systematically investigated.

The sample used in this study was grown by the twoflow metal-organic chemical-vapor deposition method, the details of which have been described elsewhere. ELO GaN was fabricated by the procedure described below. First, a

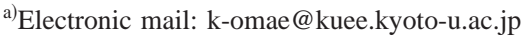

$2-\mu \mathrm{m}$-thick GaN layer was grown on a (0001) $C$-face sapphire substrate. The $0.1-\mu \mathrm{m}$-thick $\mathrm{SiO}_{2}$ mask was patterned to form 4- $\mu \mathrm{m}$-wide stripe windows with a periodicity of 12 $\mu \mathrm{m}$ in the GaN $\langle 1-100\rangle$ direction. The structure is then completed by the $10-\mu$ m-thick $\mathrm{GaN}$ growth on the $\mathrm{SiO}_{2}$ mask pattern. A flat $\mathrm{GaN}$ surface over the entire substrate is achieved as a result of the coalescence of the selectively grown GaN. Threading dislocation densities were roughly estimated to be $10^{6} \mathrm{~cm}^{-2}$ in the wing region $(\mathrm{GaN}$ grown on the $\mathrm{SiO}_{2}$ mask), and $10^{8} \mathrm{~cm}^{-2}$ in the window region. The excitation sources used were a $\mathrm{He}-\mathrm{Cd}$ laser $(325 \mathrm{~nm})$ for continuous-wave (cw) photoluminescence (PL), and a thirdharmonic beam of a mode-locked $\mathrm{Al}_{2} \mathrm{O}_{3}$ :Ti laser (wavelength: $266 \mathrm{~nm}$, pulse width: $1.5 \mathrm{ps}$, repetition rate: $80 \mathrm{MHz}$ ) or a frequency dually doubled beam $\left(4 \omega_{s}\right)$ of an optical parametric amplifier (OPA) (wavelength: $330 \mathrm{~nm}$, pulse width: $150 \mathrm{fs}$, repetition rate: $1 \mathrm{kHz}$ ) for the pulsed PL. The FWM measurements were performed in the standard twopulse self-diffraction configuration in transmittance geometry using the $4 \omega_{s}$ beam of the OPA in the range of 354-364 $\mathrm{nm}$ with an excitation energy density of $200 \mu \mathrm{J} / \mathrm{cm}^{2}$. The external-crossing angle of the two beams is below $10^{\circ}$. The whole spectra were taken at $10 \mathrm{~K}$.

Figure 1 shows the reflection and the cw PL spectra of the sample. By comparison between the two, PL peaks at 3.4945 and $3.5025 \mathrm{eV}$ are attributed to the $A$ free exciton $\left(E_{X A}\right)$ and the $B$ free exciton $\left(E_{X B}\right)$, respectively. These energy positions are shifted towards the higher photon energy side by about $17 \mathrm{meV}$ compared to that of the nearly strainfree homoepitaxial $h$-GaN layer. ${ }^{6}$ This is because the epilayer suffers biaxial compression of the order of $13 \mathrm{kbar}$ at the face perpendicular to the (0001) direction, which is mainly induced by the difference of the thermal expansion coefficient between the substrate and the epilayer. The main PL peak located at $3.4880 \mathrm{eV}$ is attributed to the excitons bound to the neutral donor $\left[\left(D^{0}, X\right)\right]$. This energy position is very close to that of biexcitons which appear under moderately high photoexcitation. ${ }^{7}$ It should be noted that the spec- 


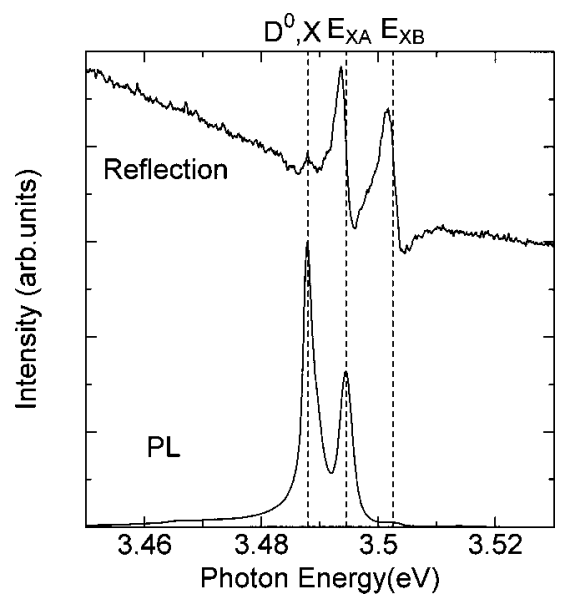

FIG. 1. PL spectrum of an ELO-GaN sample at $10 \mathrm{~K}$ under cw excitation with a $\mathrm{He}-\mathrm{Cd}$ laser. Reflection spectrum is also inserted in the figure.

tra in Fig. 1 are taken under macroscopic condition where a lot of stripes composed of wing/window regions are included within the area of photoexcitation. In order to assess the correlation between dislocation densities and optical properties, the microscopic PL was performed under the siteselective configuration. It was found that the spectral shape of the spectra taken at the wing region is almost the same as that at the window region, besides the former is blueshifted by $0.3 \mathrm{meV}$ with respect to the latter due to a slight difference of compressive strain. This suggests that the optical quality is insensitive to macroscopic dislocation densities, and that the transition energies can be determined within the accuracy of $0.3 \mathrm{meV}$ even with a macroscopic measurement.

Figure 2(a) shows the picture of transmitted laser beams used for the FWM measurement, the directions of which are defined as $\mathbf{k}_{1}$ and $\mathbf{k}_{2}$, respectively. Since the striped $\mathrm{SiO}_{2}$ mask acts as a grating, each beam is diffracted to the direction perpendicular to the periodic structures of an ELO GaN. The wavelength is $357 \mathrm{~nm}(3.473 \mathrm{eV})$, so that the incident beams transmit the sample with almost no photoabsorption. If the timings of the two beams are tuned, FWM signals appear in the phase matching directions, showing third $\left(2 \mathbf{k}_{2}-\mathbf{k}_{1}\right)$, fifth $\left(3 \mathbf{k}_{2}-2 \mathbf{k}_{1}\right)$, seventh $\left(4 \mathbf{k}_{2}-3 \mathbf{k}_{1}\right)$, ninth $\left(5 \mathbf{k}_{2}-4 \mathbf{k}_{1}\right)$, eleventh $\left(6 \mathbf{k}_{2}-5 \mathbf{k}_{1}\right)$, thirteenth $\left(7 \mathbf{k}_{2}-6 \mathbf{k}_{1}\right)$, and fifteenth $\left(8 \mathbf{k}_{2}-7 \mathbf{k}_{1}\right)$-order nonlinearlity, as shown in Fig. 2(b). To the naked eye, signals up to eleventh-order
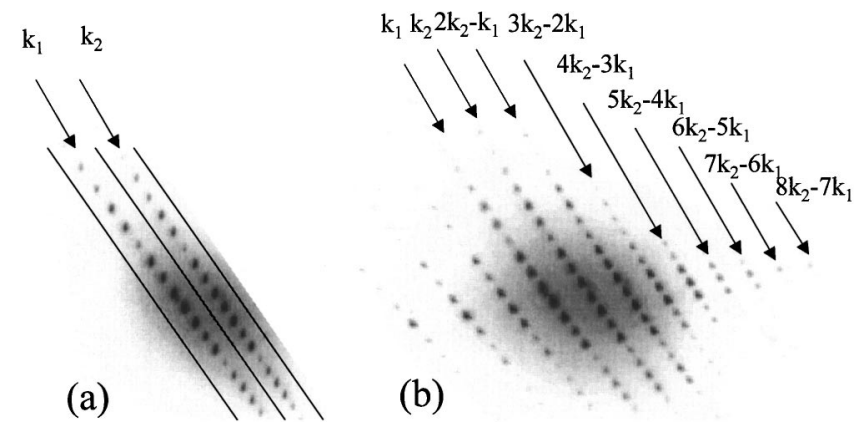

FIG. 2. (a) Photograph of transmitted two-pump beams in the direction of $\mathbf{k}_{1}$ and $\mathbf{k}_{2}$ used for FWM experiments. The timing of the two beams are detuned to each other. Each beam is diffracted to the direction perpendicular to the periodic striped structures of an ELO-GaN. (b) Photograph of the wave mixing signals at $10 \mathrm{~K}$. The incident photon energy is $3.473 \mathrm{eV}$ with

excitation energy density of $200 \mu \mathrm{J} / \mathrm{cm}^{2}$.
Downloaded 04 Mar 2008 to 130.54 .110 .22 . Redistribution subject to AlP license or copyright; see http://apl.aip.org/apl/copyright.jsp
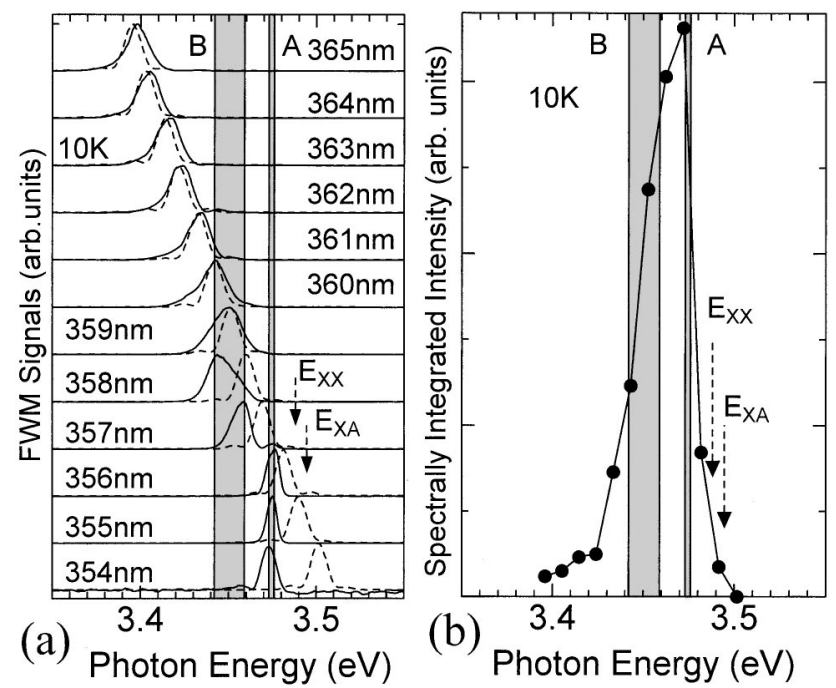

FIG. 3. (a) FWM spectra (solid curve) at $10 \mathrm{~K}$ taken under various pumping spectral conditions (dotted curve). (b) Spectrally integrated FWM intensities as a function of excitation energy. Energy positions of $E_{X A}$ and $E_{X X}$ are

nonlinearlity can be observed. If the incident energy density is less than about $20 \mu \mathrm{J} / \mathrm{cm}^{2}$, such optical nonlinearlity is not observed at energies below the fundamental absorption edge. It should be noted that similar high-order nonlinearlities have been observed in ZnSe-based semiconductors. ${ }^{8,9}$ The finding of optical nonlinearlity at the transparent energy range is interesting not only from the physics viewpoint but also from applications related to the high-speed multisignal processing with low optical conditions.

The FWM spectra at $10 \mathrm{~K}$, taken under various pumping spectral conditions, are shown in Fig. 3. Each signal is measured at each time $(t=0-275 \mathrm{fs})$ when the maximum intensity is reached. Unlike the case under lower photoexcitation energy density, the nonlinear signals originating from the transitions of $E_{X B}, E_{X A}$, and $E_{X X}$ are not detected in this condition probably because the carrier density exceeds the Mott's screening density of excitons. FWM signals are pinned instead at two energy ranges, labeled $A$ (3.473-3.476 $\mathrm{eV})$ and $B(3.442-3.459 \mathrm{eV})$ if the incident is covered beam in the vicinity of these energy positions. Since the absorption tail in GaN is negligibly small in such energy ranges at cryogenic temperature, almost no real exciton population is involved in these nonlinear processes. Moreover, if the incident laser wavelength is in the range of $354-358 \mathrm{~nm}$, the time when the maximum intensity is reached is delayed with respect to that of the laser pulse. The spectra taken under such wavelength conditions are pinned at the energy positions of $A$ and $B$. From these results, the origin of the $A$ - and $B$-nonlinear bands in the FWM spectra may be ascribed to virtual excitonic states related to the many-body effect of excitons such as exciton-exciton scattering or to excitonphonon complex systems.

The spectrally integrated intensities of the FWM signals as a function of pumping laser energies are shown in Fig. 3(b). The maximum intensity is obtained at the pumping energy of $3.473 \mathrm{eV}$, which is almost the same as the energy position of $A$. However, it should be noted that the peak of the FWM spectrum jumps to the $B$ range if the incident laser energy is tuned to peak $A$, as shown in the spectrum in Fig. AIP license or copyright; see http://apl.aip.org/apl/copyright.jsp shown for the reference. 


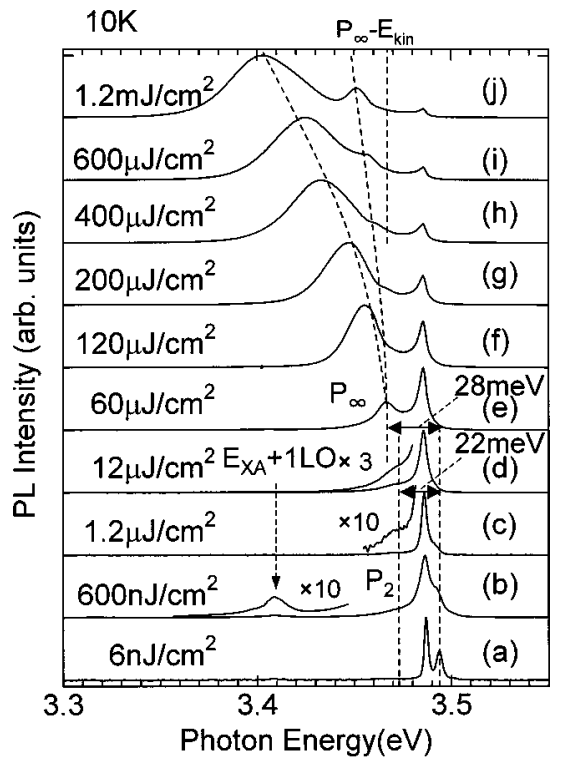

FIG. 4. Time-integrated PL spectra of ELO-GaN at $10 \mathrm{~K}$ taken under pulsed excitation with different excitation energy densities. For excitation energy density less than $600 \mathrm{~nJ} / \mathrm{cm}^{2}$, the excitation source is the third harmonic beam of a mode-locked $\mathrm{Al}_{2} \mathrm{O}_{3}$ :Ti laser, and is the frequency dually-doubled beam of an optical parametric amplifier above $1.2 \mu \mathrm{J} / \mathrm{cm}^{2}$.

3(a) taken under $357 \mathrm{~nm}$ excitation. It is likely that a complicated interaction between the light field and virtual exciton states plays a role for this anomalous behavior.

In order to identify the energy positions of the optical transitions related to dense excitons, time-integrated PL (TIPL) spectra were detected as a function of increasing pump intensity, as depicted in Fig. 4. The excitation energy density ranged from $6 \mathrm{~nJ} / \mathrm{cm}^{2}$ to $1.2 \mathrm{~mJ} / \mathrm{cm}^{2}$. The TIPL spectrum under $I_{\mathrm{ex}}=6 \mathrm{~nJ} / \mathrm{cm}^{2}$ is very similar to that under the $\mathrm{cw}$ excitation (Fig. 1). The intensity of the $\left(D^{0}, X\right)$ band tends to saturate with increasing $I_{\text {ex }}$ up to about $60 \mathrm{~nJ} / \mathrm{cm}^{2}$, and then grows if the $I_{\mathrm{ex}}$ value is further increased. It is probable that the saturation is due to the filling of shallow donor centers, and that the growth is contributed from biexcitons $\left(E_{X X}\right)$ as reported before. Above about $I_{\mathrm{ex}}=1.2 \mu \mathrm{J} / \mathrm{cm}^{2}$, a new emission peak appears at about $3.473 \mathrm{eV}$. The energy difference between this peak and $E_{X A}$ is about $22 \mathrm{meV}$. This value is almost the same as three quarters of the exciton binding energy $\left(3 / 4 E_{\text {ex }}\right)$. Therefore, this new emission peak can be assigned to the $P_{2}$ transition, where two excitons are converted to a photon-like state and an excited state of an exciton from $1 S$ to $2 S .{ }^{10,11}$ The energy position of this transition is nearly equal to the $A$ range observed in FWM spectroscopy. The $P_{2}$ emission is replaced by the band peaked at $3.467 \mathrm{eV}$, as seen in the spectrum under $I_{\mathrm{ex}}=60 \mu \mathrm{J} / \mathrm{cm}^{2}$. Considering its energy position $\left(E_{X A}-P_{\infty}=E_{\text {ex }}\right)$, it is natural to ascribe this band to $P_{\infty}$, where one remaining exciton in the $P_{2}$ is dissociated to the free electron and hole in this process. ${ }^{12}$ Further increasing excitation energy density, the $P_{\infty}$ bands are redshifted by $\delta E_{\text {kin }}$, because they are scattered to higher-kinetic-energy states, satisfying momentum and energy conservation rules. It was found that $\delta E_{\text {kin }}$ changes with the relation of $\delta E_{\mathrm{kin}} \propto I_{\mathrm{ex}}^{1 / 3}$. This is consistent with the theoretical prediction in the case of three-dimensional bulk material considering the density of states of carriers involved in this scattering process. ${ }^{13}$ Above about $I_{\mathrm{ex}}=60 \mu \mathrm{J} / \mathrm{cm}^{2}$, the $P_{\infty}$ band branches also to the lower-energy band. This emission band becomes broader with increasing $I_{\mathrm{ex}}$ values and their peak energies make redshifts strongly. Therefore, these bands are probably attributed to radiative recombination of the electron-hole plasma (EHP),${ }^{14}$ where Coulomb attractive forces between electrons and holes are completely screened by the large amount of carriers. Under $I_{\mathrm{ex}}=1.2 \mathrm{~mJ} / \mathrm{cm}^{2}$, the peak energy of the EHP band reaches $3.405 \mathrm{eV}$, which is nearly the same as that of the $E_{X A}+1 \mathrm{LO}$ (longitudinal optical phonon) band (3.409 eV) (see, also, Fig. 4).

As mentioned before, the origin of the $A$-nonlinear band in the FWM spectra may be related to virtual excitonexciton $P_{2}$ scattering comparing with FWM and PL spectra. However, no clear correspondence was observed between the $B$-nonlinear band and PL peak energies. This $B$ band (3.442$3.459 \mathrm{eV}$ ) is located between $P_{2}$ and the $E_{X A}+1 \mathrm{LO}$ bands. It may be related to three or more exciton scattering processes, or other complicated many-body processes where excitons, biexcitons, and free carriers are involved. However, it is difficult at the current stage to make a clear assignment of the $B$ band. This finding is important for the future development of nonlinear optical switching devices operated under transmittance geometry with low optical loss conditions.

The authors thank Dr. Narukawa at Nichia Corporation for fruitful comments and encouragement. The authors are also grateful for financial support of the Grant-in-Aid for Scientific Research from the Ministry of Education, Culture, Sports, Science and Technology. A part of this work was performed using the facility at the Venture Business Laboratory in Kyoto University (KU-VBL).

${ }^{1}$ A. J. Fisher, W. Shan, G. H. Park, J. J. Song, D. S. Kim, D. S. Yee, R. Horning, and B. Goldenberg, Phys. Rev. B 56, 1077 (1997).

${ }^{2}$ R. Zimmermann, A. Euteneuer, J. Möbius, D. Weber, M. R. Hofmann, W. W. Rühle, E. O. Göbel, B. K. Meyer, H. Amano, and I. Akasaki, Phys. Rev. B 56, R12722 (1997).

${ }^{3}$ T. Aoki, G. Mohs, T. Ogasawara, M. Kuwata-Gonokami, and A. A. Yamaguchi, Phys. Rev. Lett. 82, 3108 (1999).

${ }^{4}$ B. Monemar, Phys. Rev. B 10, 676 (1974).

${ }^{5}$ S. Yu, J. H. Chu, J. I. Lee, D. Kim, Y. H. Yee, D. S. Kim, J.-Y. Leem, C.-R. Lee, and J. H. Lee, Appl. Phys. Lett. 69, 79 (1996).

${ }^{6}$ K. Pakula, A. Wysmolek, K. P. Korona, J. M. Baranowski, R. Stepniewski, I. Grzegory, M. Bockowski, J. Jun, S. Krukowski, M. Wroblewski, and S. Porowski, Solid State Commun. 97, 919 (1996).

${ }^{7}$ Y. Kawakami, Z. Peng, Y. Narukawa, Sz. Fujita, Sg. Fujita, and S. Nakamura, Appl. Phys. Lett. 69, 1414 (1996).

${ }^{8}$ A. J. Fischer, D. S. Kim, J. Hays, W. Shan, J. J. Song, D. B. Eason, J. Ren, J. F. Schetzina, H. Luo, J. K. Furdyna, Z. Q. Zhu, Y. Tao, J. J. Klem, and W. Schäfer, Phys. Rev. Lett. 73, 2368 (1994).

${ }^{9}$ D. S. Kim, A. J. Fischer, J. Hays, W. Shan, J. J. Song, D. B. Eason, J. Ren, and J. F. Schetzina, Appl. Phys. Lett. 65, 1534 (1994).

${ }^{10}$ L. Eckey, J. Holst, A. Hoffmann, I. Broser, T. Detchprohm, and K. Hiramatsu, MRS Internet J. Nitride Semicond. Res. 2, 1 (1997).

${ }^{11}$ S. Kurai, A. Kawabe, T. Sugita, S. Kubo, Y. Yamada, T. Taguchi, and S. Sakai, Jpn. J. Appl. Phys., Part 2 38, L102 (1999).

${ }^{12}$ T. J. Inagaki, M. Aihara, and A. Takahashi, Solid State Commun. 115, 645 (2000).

${ }^{13}$ P. R. Newbury, K. Shahzad, and D. A. Cammack, Appl. Phys. Lett. 58, 1065 (1991).

${ }^{14}$ J. Holst, L. Eckey, A. Hoffmann, I. Broser, T. Detchprohm, H. Amano, and I. Akasaki, MRS Internet J. Nitride Semicond. Res. 2, 25 (1997). 